\title{
Paul Davies and Why Time Is Not a Flow
}

\section{Vincent Vesterby}

\section{PART ONE: Time, Flow, and the River Analogy}

When John Steele interviewed Paul Davies on the subject of time (Steele 2014), Davies used an analogy with a flowing river to explain why time is not a flow. He was correct, time does not flow. Davies' reasoning, however, was flawed, and did not actually explain why time does not flow.

The analogy between time and a flowing river probably originated because it was recognized that both time and the river are cases of continuous ongoing change.

With the time part of the analogy, time is flowing past the present.

With the flowing river part of the analogy, there is the river flowing along between its banks. There is a specific place on the bank, usually with a person standing there watching the river flow by. The place where the person is standing represents the present, where the water is currently flowing by. The water upstream represents the future because it has not yet reached the person on the bank. The water downstream from the person represents the past because it has already passed by the person.

This analogy is clear-the water flows with respect to a specific location on the bank which represents the present.

Davies gives a confused version of the analogy.

Paul Davies: The flow of time is an illusion, ... The reason that it is an illusion is when you stop to think, what does it even mean that time is flowing? When we say something flows like a river, what you mean is an element of the river at one moment is in a different place of an earlier moment. In other words, it moves with respect to time. But time can't move with respect to time- - time is time.

The objective of Davies' argument is to remove flow from the understanding of the change that occurs with time, which is a valid objective. His argument is flawed because in his attempt to remove flow from time, he uses language that removes flow from the river part of the analogy. He tries to remove flow from time by trying to remove flow from the river.

Davies says "When we say something flows like a river, what you mean is an element of the river at one moment is in a different place of an earlier moment."

That is not what the term, flow, means. When something is flowing, it is moving. With the river, it is water moving with respect to the river channel, with respect to the river 
banks. With flow it is not that something is in one place at one moment, and in another place at a different moment, but that it moves from place to place, it flows from place to place.

That 'an element of the river' is in a different place from moment to moment is a consequence of the flow, a consequence of the physical motion. Davies' language, ". . . an element of the river at one moment is in a different place of an earlier moment", leaves out the relevant factor of how the "element of the river" gets from place to place from moment to moment-it flows.

While the water does flow with respect to time, that is not the point of the analogy. In the analogy it is not that the water of the river moves with respect to time, but that it moves with respect to a specific location on the bank of the river.

When Davies said, "In other words, it [the water] moves with respect to time," that statement confuses the point of the analogy. But it does give Davies the opportunity to say, "But time can't move with respect to time - time is time." This sophistry is Davies' way to eliminate the concept of time as a flowing form of change-which does not actually work.

The problem here is that Davies does not know what time is, why time occurs, or why it has the specific qualities (properties) that it has, such as what kind of change time is. He does not know what constitutes the basis of time in the universe. The important point here is that it is necessary to know what kind of change time is in order to know why time is not a flow.

The river analogy itself introduces a significant confusion because the ongoing change of flowing involves the movement of matter, but the ongoing change of time does not involve matter or motion. Flow and time are significantly different forms of change.

\section{PART TWO: Why Time Is Not a Flow Section 1: Finding Time}

No one, down through the ages, has ever known what time is, why it exists, or why it has the specific qualities that it has. While some of the qualities of time have been identified - it is a form of change and that change is unidirectional - the basis of those qualities has never been identified. All discussion of the intrinsic nature of time has, of necessity, been speculation. Recognition of the basis of time in the universerecognizing what time is - removes the need for speculation, and makes it possible to understand what kind of change time is.

The use of the type of transdisciplinary methodology that results in multi-disciplinespanning understanding and that enhances communication among the disciplines revealed the basis of time in the universe from a bottom-up approach. Using transdisciplinary methodology to investigate the intrinsic nature of space revealed that the intrinsic qualities of the continuing-existence of space are the same as the qualities that can be realistically attributed to time. It became evident that spatial-continuing-existence plays 
all the roles in the universe that are commonly thought of as temporal roles. (Vesterby 2019)

(This is the continuing-existence of space as measured by a clock, not the extension of three-dimensional space as measured by a ruler.)

Spatial-continuing-existence was being studied and described before it was realized that it was the basis of time. When the realization occurred that spatial-continuing-existence was the basis of time, it became evident why the foundation of time in the universe had not been previously recognized. The top-down approach of viewing the known qualities of time does not lead to the understanding that spatial-continuing-existence is that foundation. The basis of time could only be discovered inadvertently, by a bottom-up approach, by studying the basis and describing it, and only then recognizing that it is the basis.

\section{Section 2: Continuances}

Once it is known what time is, what form of ongoing change it is, then it is possible to understand why time is not a flow.

Spatial-continuing-existence is the form of change by which time occurs. So, what kind of change is continuing-existence? Why is continuing-existence a form of change? This is a form of change that is not much discussed.

(The following discussion is not about concepts. It is about the reality-referents of concepts. It is not about the concepts of space or spatial-continuing-existence. It is about space itself and spatial-continuing-existence as they exist as intrinsic qualities of the universe. Concepts are recognized to be mental tools that are used by the mind to achieve understanding of the world outside the mind. The discussion avoids abstraction and all forms of speculation from presuppositions and assumptions to hypotheses and theories.)

The human visual sense has evolved such that it can detect the presence of space. When looking at space, it can be seen that space is there, and that it continues to be there. It can be observed that spatial-continuing-existence is a continuance of being-there. Continuingexistence is a form, a type, of continuance.

\section{Section 3: Parts}

All forms of continuance have parts, for example, a limited form of continuance has a beginning part, a middle part, and a final part, as occurs with a broom handle from the upper end down to the lower end, or as occurs with a single rotation of the earth.

The parts of a continuance occur sequentially, each part occurring after the previous part and before the following part.

\section{Coexistent-Sequential-Difference}

With the case of the broom handle, all the sequential parts are there together-it is possible to see them all together at the same time, the full length of the handle. Each part 
is individually unique — the upper part, the middle part, and the lower part — each sequentially different from a following part or from a prior part. With the broom handle, the parts are sequentially different, coexistent, and constitute a situation of coexistentsequential-difference.

\section{Noncoexistent-Sequential-Difference}

With the case of the rotating earth, the sequentially occurring parts of a rotation are not there together - it is not possible to see them all together at the same time, the full rotation from beginning to end. Each part of the rotation - the first part, the middle part, and the final part—is individually unique, sequentially different from a following part and from a prior part. With the rotation of the earth, the parts are sequentially different, are not sequentially coexistent, and constitute a situation of noncoexistent-sequentialdifference.

\section{Section 4: No Change-Change}

With the coexistent-sequential-difference that exists along the handle of a broom, there is difference from place to place, but there is no occurrence of change.

With the noncoexistent-sequential-difference that occurs from part to part with rotation, the difference that occurs does so in the form of change.

As with rotation, flow and spatial-continuing-existence are cases of noncoexistentsequential-difference in which the difference that occurs does so in the form of change.

Section 5: New Part of Noncoexistent-Sequential-Difference

When observing the broom handle - the full length of it, all the coexistent parts simultaneously - there is no ontological difference occurring, no existential changes occurring. All the parts are sequentially there together, and they remain there together.

When observing rotation, flow, and the continuing-existence of space- the noncoexistent sequence of the parts - ontological difference occurs, existential change occurs. The parts occur noncoexistently, and are not there together. When the current part is there, the previous part no longer exists and the following part has not yet come into existence. The part of the rotation, the flow, or the continuing-existence of space that is occurring currently is noncoexistently distinct from the part that occurred just previous. The current part of their noncoexistent-sequential-difference did not yet exist when the previous part was occurring.

Now, as the current part exists, it is newly existent. As the rotation continues, as the flow continues, as space continues to exist, there is continuously new part of those cases of ongoing continuance - new part of ongoing noncoexistent-sequential-difference-new part of the ongoing rotation, new part of the ongoing flow, new part of the continuingexistence of space.

Change is the occurrence of something which is existentially new-the coming into existence of something that is existentially new. 


\section{Section 6: Basis of New Part}

The occurrence of new part of noncoexistent-sequential-difference is the occurrence of change. With the cases of rotation and flow it is new part of ongoing motion of matterrotating matter of the earth and flowing water along the river channel. Space, however, is immaterial. As a foundational component of the universe, space exists as the infinite three-dimensional extension of immaterial place.

(The attribution of a material basis for space is anthropomorphism-humans have a material basis. The attribution of any quality or property of substantiality or matter to space is also anthropomorphic. Anthropomorphism is disallowed in science and the philosophy of reality, the philosophy of that which exists.)

Neither matter nor motion play any roles in the existence or qualities of space or in the continuing-existence of space. The basis of the noncoexistent-sequential-difference of spatial-continuing-existence is different from the basis of the noncoexistent-sequentialdifference of flow and rotation. The intrinsic nature of new part of spatial-continuingexistence is different from the nature of new part of the ongoing motion of matter.

\section{Section 7: Distinction Between Flow and Spatial-Continuing-Existence}

Both flow and spatial-continuing-existence are cases of continuous ongoing change. Both are cases of noncoexistent-sequential-difference. Both have the occurrence of new part of the continuous ongoing change, new part of their noncoexistent-sequential-difference.

They are different in what it is that constitutes the continuous ongoing change. With flow it is ongoing motion, which involves a role for matter-it is matter that moves. With spatial-continuing-existence it is continuing-existence, which involves a role for spacespace exists and continues to exist.

Flow and spatial-continuing-existence are different in what constitutes new part. With flow it is new part of ongoing motion. With spatial-continuing-existence it is new part of that ongoing existence.

\section{Section 8: The Relation between Time and Flow}

Existing as the infinite three-dimensional extension of immaterial place, space provides an existential-context, a place-to-be, a place in which to exist, for all else that exists. The three-dimensional extension of immaterial spatial-place provides an existential-context for the three-dimensional extension of matter.

Spatial-continuing-existence provides an existential-context, a place-to-occur, for all other forms of change. Spatial-continuing-existence provides an existential-context for the continuing-existence of matter - the continuing-existence of an object occurs concurrently, simultaneously, with the continuing-existence of the spatial-place the matter occupies. 
The continuous ongoing change of spatial-continuing-existence provides an existentialcontext for the continuous ongoing change that occurs with motion, that occurs with flow. The relation between time and flow is that time provides the existential-context in which flow occurs. By providing an existential-context for all forms of change, spatialcontinuing-existence plays throughout the universe all the roles of time.

\section{PART THREE: Transdisciplinarity, Isomorphies, and Temporal Change}

This discussion about the nature of the change of time is based on transdisciplinary methodology. This methodology is based on patterns-of-organization of structure and process that occur again and again throughout the subject matters of the various disciplines. These ubiquitous patterns-of-organization are called isomorphies, and knowledge of them is used to enable understanding that spans the disciplines.

An isomorphy has a basic form which is there in every situation in which the isomorphy plays a role-in every discipline in which that particular isomorphy occurs. While the basic pattern of an isomorphy is identifiable in every situation in which it occurswhether it is a pattern of relations between molecules or a pattern of relations between members of a social group - the nature of an isomorphy and the roles it plays are influenced by the other factors playing roles in the situation in which the isomorphy exists.

Sequential-difference is an isomorphy. The different forms it has in different situations was used to clarify why time is not a flow. In each situation there were other factors that influenced the nature of sequential-difference in those situations.

With the broom handle, sequential-difference occurs as coexistent-sequential-difference because it is based on the stable coherent structure of the wood or plastic of the handle. All the sequentially related parts of the broom handle exist together, concurrently, simultaneously, without any role for change. In this situation the sequential-difference is based on matter.

With the motion of the rotating earth, sequential-difference occurs as noncoexistentsequential-difference because it is based on the motion of the rotation. With the occurrence of motion, the parts of the ongoing motion are not all there together. The parts of motion do not occur concurrently, simultaneously. Instead, as the parts of an ongoing motion occur, the previous parts no longer exist and the following parts have not yet occurred. In this situation the sequential-difference is based on the motion, but there is still a role for matter because it is matter that moves.

With the continuing-existence of space, sequential-difference again occurs as noncoexistent-sequential-difference, but in this situation, it is because it is based on the ongoing existence of space. With the occurrence of ongoing existence, the parts are not all there together. The parts of continuing-existence do not occur concurrently, simultaneously. As the parts of continuing-existence occur, the previous parts no longer exist and the following parts have not yet occurred. In this situation the sequentialdifference is based on immaterial space, without any role for matter or motion. 
In each of these situations the sequential-difference is based on different factors, occurs in different forms, and plays different roles.

\section{REFERENCES}

Steele, John. 2014. Where Did Time Come From, and Why Does It Seem to Flow? Nautilus. Jan 28, 2014

http://nautil.us/blog/where-did-time-come-from-and-why-does-it-seem-to-flow

Vesterby, Vincent. 2019. "The Identification of the Intrinsic Nature of Time.” OSF Preprints. July 26. osf.io/gqxjy. https://osf.io/gqxjy

Vesterby, Vincent. 2012. Discipline-Independent-Transdisciplinarity: The Essentials. https://www.researchgate.net/publication/299397879_Discipline-IndependentTransdisciplinarity_The_Essentials

Here are two other papers that provide additional understanding about the intrinsic nature of time.

Vesterby, Vincent. 2019. “The Basis of Time in the Universe.” OSF Preprints. July 24. osf.io/q4ntk. https://osf.io/q4ntk

Vesterby, Vincent. 2019. "Temporal Naturalism—analysis of the Paper by Lee Smolin." OSF Preprints. July 29. osf.io/mk9wz. https://osf.io/mk9wz

Date of original version of "Paul Davies and Why Time Is Not a Flow"-2016. 